\title{
Alternative Developer Solutions and Processes for EUV and ArFi Lithography
}

\author{
Masahiko Harumoto ${ }^{1}$, Julius Joseph Santillan ${ }^{2 *}$, Chisayo Nakayama ${ }^{1}$, Yuji Tanaka ${ }^{1}$, \\ Tomohiro Motono ${ }^{1}$, Masaya Asai ${ }^{1}$, and Toshiro Itani ${ }^{2}$ \\ ${ }^{1}$ SCREEN Semiconductor Solutions Co., Ltd., \\ 480-1 Takamiya-cho, Hikone, Shiga 522-0292, Japan \\ ${ }^{2}$ The Institute of Scientific and Industrial Research, Osaka University, \\ 8-1 Mihogaoka, Ibaraki, Osaka 567-0047, Japan \\ *julius.santillan@sanken.osaka-u.ac.jp
}

\begin{abstract}
The application of alternative developers for both extreme ultraviolet (EUV) and 193-nm immersion ("ArFi") lithography is investigated by focusing on their effects on the "DefectNot-Found" $(D N F)$ margins. In case of EUV lithography, defects primarily comprise line bridging at the underdose region and line breaks at the overdose region. The application of a $0.26-\mathrm{N}$ tetrabutylammonium hydroxide (TBAH) developer solution when compared to that of a $0.26-\mathrm{N}$ tetramethylammonium hydroxide (TMAH) developer solution resulted in improved resist sensitivity while maintaining the same DNF margin. In case of ArFi lithography, defects primarily comprise line bridging at the underdose region and pattern collapse at the overdose region. The same improvement that has been mentioned above can be observed with respect to the resist sensitivity using the TBAH developer solution. However, the TBAH developer solution significantly minimizes the pattern collapse at the overdose region, further extending the minimum line pattern size. This translated to significant improvements in both the exposure latitude $(E L)$ and overdose margin $(O M)$ and resulted in a significant increase in the DNF margin. Furthermore, the application of a nonionic surfactant type additive on the TMAH developer solution demonstrates resist sensitivity improvement and pattern collapse mitigation. Increased $E L$ and $O M$ values were observed, which also resulted in increased DNF margin. Results indicate the advantages of utilizing alternative developer solutions in improving the DNF margins.
\end{abstract}

Keywords: Developer solution, TMAH, TBAH, EUV lithography, ArFi lithography

\section{Introduction}

Ongoing and successful efforts in the development of lithographic technologies continue to be crucial to further extend the scaling targets for semiconductor devices [1-3]. 193-nm immersion ("ArFi") and the recent extreme ultraviolet (EUV) lithography are the frontrunners in achieving critical patterning targets [1,2]. Resist materials are important in achieving the targeted resolution limits [4-6]. Here, advancement and, at times, drastic changes in resist materials have been performed to adapt and obtain the set targets [7-9]. However, currently, lithography is entering a generation of patterning, where resolution and stringent control of resist-based defects (e.g., residue, line bridge, line break, and pattern collapse) are of utmost importance and must be prioritized [6,10-13]. Therefore, improving the resist processes is considered to be a potential solution.

This study explores the application of alternative resist processes in the form of developer solutions for both EUV and ArFi lithography resist materials.

\section{Materials and processing}

2.1. EUV lithography materials and processing

A chemically amplified resist (CAR) was utilized at a film thickness of $30 \mathrm{~nm}$ under optimal post application bake (PAB) and post exposure bake (PEB) conditions. The EUV CAR (typically a 
hybrid of polyhydroxystyrene- and acryl-based polymers) was coated onto a $300-\mathrm{mm}$ wafer coated with an organic underlayer at a thickness of $20 \mathrm{~nm}$. The development process after PEB was performed at a development time of $30 \mathrm{~s}$ using several types of developer solutions as described in 2.3. The rinse process was performed using deionized water with a rinse time of approximately $19 \mathrm{~s}$.

The resist processes from coating up to PEB were performed using the SOKUDO DUO coater/developer (SCREEN Semiconductor Solutions) at IMEC, Belgium. The development process was performed using the RFi coater/developer (SCREEN Semiconductor Solutions) in Hikone, Japan. The EUV patterning exposures were performed using the NXE-3300 (ASML) with a numerical aperture (NA) of 0.33 under optimal illumination conditions. All the SEM measurements were performed using CG5000 (Hitachi High Technologies).

\subsection{ArFi lithography materials and processing}

Another type of CAR was utilized (film thickness: $130 \mathrm{~nm}$ ) under optimal PAB and PEB conditions. The ArFi CAR (typically acryl-based polymer) was coated onto a $300-\mathrm{mm}$ wafer that was coated with a film stack of silicon hardmask $(40 \mathrm{~nm})$ and spin-on carbon $(200 \mathrm{~nm})$. The development process after PEB was performed with a development time of $60 \mathrm{~s}$ using several types of developer solutions. The rinse process was performed using deionized water with a rinse time of approximately $30 \mathrm{~s}$. Note that the CAR film thickness was $30 \mathrm{~nm}$ thicker than the typical application conditions to realize accelerated testing for pattern collapse.

The resist processes from coating up to PEB were performed using a clean track coater/developer at the National Institute of Advanced Industrial Science and Technology (AIST) super clean room (SCR) facility in Tsukuba, Japan. Note that the ArFi lithographic patterning exposures were performed using the 1.3 NA NSR-S610C (Nikon) under optimal illumination conditions in the same facility. The development process was performed with a clean track coater/developer at the same facility. All the SEM measurements were performed using CG4000 (Hitachi High Technologies).

\subsection{Developer solutions}

Figure 1 denotes two types of aqueous developer solutions selected for both EUV and ArFi application. The tetramethylammonium hydroxide
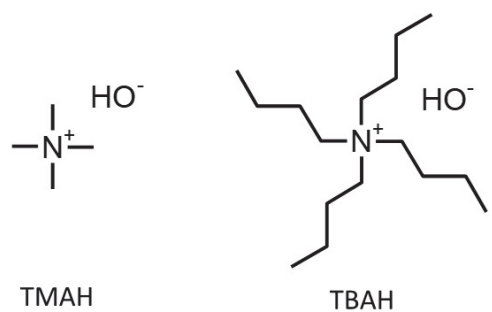

TBAH

Fig. 1. Selected aqueous alkali developer solutions.

(TMAH) developer solution, the present de facto standard in the lithography community, was utilized at a concentration of $0.26 \mathrm{~N}$. Tetrabutylammonium hydroxide (TBAH; also utilized at a concentration of $0.26 \mathrm{~N}$ ) was selected based on early reports describing its potential for minimizing the postdevelopment process defects (e.g., pattern collapse) [14-18]. The TMAH developer solution had a contact angle (CA) of $79^{\circ}$ as tested on the resist film surface. The TBAH developer solution had a CA of $66^{\circ}$ (CA indicates the hydrophilicity or wettability of the developer solution on the resist film surface).

In addition, the application of a non-ionic type surfactant additive for the TMAH developer solution was explored for ArFi. In the past, surfactants have been used to improve the resist dissolution efficiency, especially for small patterns. This can be realized by increasing the wetting property of the developer to the resist surface $[19,20]$. For these investigations, the surfactant used was added at a concentration of $0.17 \mathrm{wt} \%$, resulting in a developer solution CA of $40^{\circ}$ when compared to the value of $79^{\circ}$ that was observed when the developer solution did not contain any additive.

\section{Results and discussion}

\subsection{EUV lithography: TMAH vs TBAH}

Figure 2 depicts the EUV lithographic performance results obtained using the TMAH and TBAH developer solutions (broken lines indicate the ultimate resolution; line width roughness (LWR) and sensitivity were obtained with the 18-nm 1:1 lines/ spaces or L/S pattern). Using the TBAH developer solution, an obvious change as observed in resist sensitivity $\left(28 \mathrm{~mJ} / \mathrm{cm}^{2}\right.$ at TBAH compared to $32 \mathrm{~mJ} / \mathrm{cm}^{2}$ at TMAH). The ultimate resolution remained at 16-nm 1:1 L/S with some evidence of bridge defects at the $15-\mathrm{nm}$ 1:1 L/S patterns. LWR was not significantly improved. A comparison of the process margin was conducted for the TMAH and TBAH developers.

Figure 3 denotes the SEM images obtained across the wafer exposed using NXE:3300 and developed using the TMAH and TBAH developer solutions. 


\begin{tabular}{|c|c|c|c|c|c|c|c|}
\hline \multirow{2}{*}{ Developer solution } & \multirow{2}{*}{$\begin{array}{l}\text { Resolution } \\
\quad(\mathrm{nm})\end{array}$} & \multicolumn{4}{|c|}{ SEM image (design size, $\mathrm{nm}$ 1:1 L/S) } & \multirow{2}{*}{$\begin{array}{l}\text { LWR } \\
(\mathrm{nm})\end{array}$} & \multirow{2}{*}{$\begin{array}{c}\text { Sensitivity } \\
\left(\mathrm{mJ} / \mathrm{cm}^{2}\right)\end{array}$} \\
\hline & & 18 & 16 & 15 & 14 & & \\
\hline TMAH & 16 & & & & & 4.5 & 32 \\
\hline TBAH & 16 & & & & & 4.2 & 28 \\
\hline
\end{tabular}

Fig. 2. EUV lithographic performance results with TMAH and TBAH developer solutions (broken lines indicate ultimate resolution. LWR and sensitivity was obtained at the $18 \mathrm{~nm} \mathrm{1:1} \mathrm{L/S} \mathrm{pattern).}$

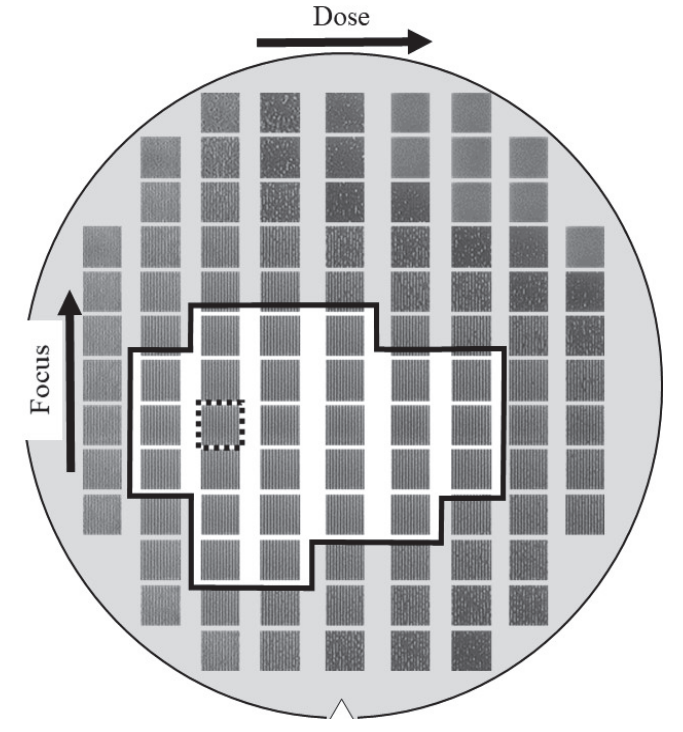

(a) TMAH

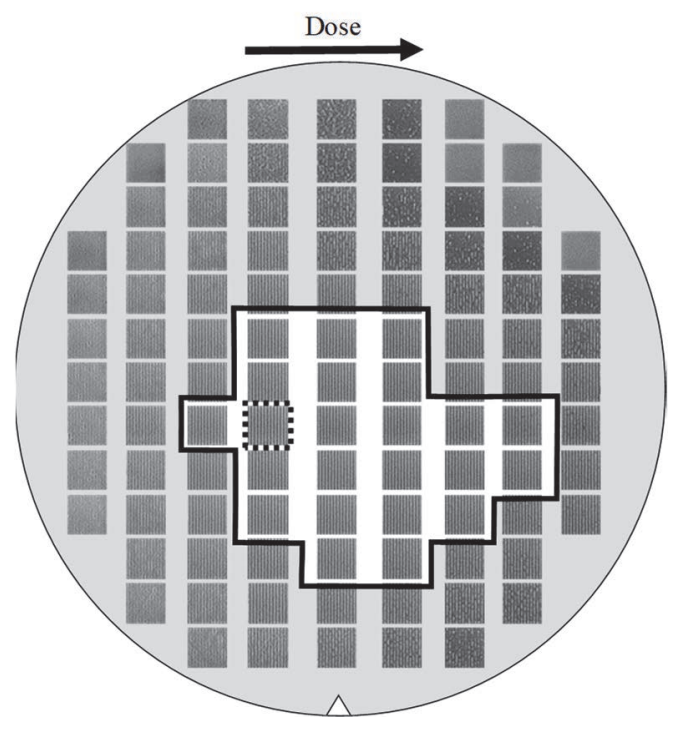

(b) TBAH

Fig. 3. SEM images obtained across the 300-mm wafer exposed using the NXE:3300 and developed using (a) TMAH and (b) TBAH developer solutions (black lines indicate the exposure area, where no line bridge or breaks were observed, and black broken lines indicate the best dose and focus condition).

These wafers were exposed as the focus-exposure matrices with dose on the $\mathrm{x}$-coordinate and focus on the y-coordinate. For the wafer developed in TMAH, the center dose was $34 \mathrm{~mJ} / \mathrm{cm}^{2}$, whereas the center dose was $30 \mathrm{~mJ} / \mathrm{cm}^{2}$ for the wafer developed in TBAH. Note that the dose steps were fixed at 2 $\mathrm{mJ} / \mathrm{cm}^{2}$. Focus conditions were done at $25 \mathrm{~nm}$ per exposure step.

In Fig. 3, the black lines indicate the exposure area, where no line bridge or breaks were observed, and the black broken lines indicate the best dose and focus condition. Subsequent analyses were

$$
\begin{aligned}
& E L=\frac{\text { Dose }_{\text {target-10\% }}-\text { Dose }_{\text {target }+10 \%}}{\text { Dose } e_{\text {target }}} \\
& O M=\frac{\text { Dose }_{<\text {break }}-\text { Dose }_{\text {target }}}{\text { Dose }_{\text {target }}}
\end{aligned}
$$

performed based on these measurement results focusing on the 18-nm 1:1 L/S pattern. Exposure latitude $(E L)$ and overdose margin $(O M)$ were calculated using Eq. (1) and (2), respectively.

where Dose target $_{\text {is }}$ the exposure dose value where measured critical dimension (CD) is equal to the target design size (in this case $18 \mathrm{~nm}$ ). Dose $_{\text {target }-10 \%}$ and Dose $_{\text {target }}+10 \%$ indicates the dose values at $-10 \%$ and $+10 \%$ of target $\mathrm{CD}$, respectively. Calculations consider and exclude exposure conditions where bridge at the underdose region and line breaks at the overdose region occur. Dose break $_{\text {is }}$ is the maximum dose before line break occurrence due to overdose.

A calculation of the "Defect-Not-Found" $(D N F)$ margin was made using Eq. (3). $C D_{<\text {bridge indicates }}$

$$
D N F=\frac{C D_{<\text {bridge }}-C D_{<\text {break }}}{C D_{\text {target }}}
$$


the largest pattern size before bridge defects occur (at the underdose region). $C D_{<\text {break }}$ shows the smallest pattern size before line breaks occur (at the overdose region). $C D_{\text {target }}$ indicates the target pattern size (in this case, $18 \mathrm{~nm}$ ).

Table 1 shows the $E L$ and $O M$ dependence on developer solution type. These results denote that a slight improvement in $E L$ was obtained using the TBAH developer solution. More significant is the increase in $O M$ with the TBAH developer solution, which indicates that the overdose window size was maintained even as the sensitivity was improved.

Table 1. EUV: $E L$ and $O M$ dependence on developer solution type.

\begin{tabular}{c|cc}
\hline $\begin{array}{c}\text { Developer } \\
\text { solution }\end{array}$ & EL (\%) & $O M(\%)$ \\
\hline TMAH & 14.6 & 24.6 \\
TBAH & 16.1 & 33.0 \\
\hline
\end{tabular}

Table 2 presents the DNF margin dependence on the developer solution. The results show that even at improved sensitivity with the TBAH when compared with that of the TMAH developer solution, $C D_{<\text {bridge }}$ and $C D_{<\text {break }}$ remained approximately the same at $\sim 20$ and $\sim 12 \mathrm{~nm}$, respectively. Thus, DNF margin remained at $\sim 40 \%$.

Table 2. EUV: DNF margin dependence on developer solution type.

\begin{tabular}{c|ccc}
\hline $\begin{array}{c}\text { Developer } \\
\text { solution }\end{array}$ & $\begin{array}{c}C D_{<\text {bridge }} \\
(\mathrm{nm})\end{array}$ & $\begin{array}{c}C D_{<\text {break }} \\
(\mathrm{nm})\end{array}$ & $\begin{array}{c}D N F \\
\text { margin }(\%)\end{array}$ \\
\hline TMAH & 20.1 & 12.8 & 41 \\
TBAH & 18.9 & 11.9 & 39 \\
\hline
\end{tabular}

Note that further investigations are required to better understand the effect of TBAH developer solutions on the EUV lithography resist performance. However, these preliminary results demonstrate that there is minimal or no disadvantageous effect on the EUV patterning capability while using the TBAH developer solution.

\subsection{ArFi lithography: TMAH vs. TBAH}

Figure 4 depicts the ArFi lithographic performance results for the 40-nm 1:1 L/S pattern obtained using the TMAH and TBAH developer solutions. As mentioned previously, to realize accelerated testing, the utilized CAR film thickness was $30 \mathrm{~nm}$ thicker than the typical application conditions, i.e., $100 \mathrm{~nm}$.

The results demonstrate the occurrence of improved sensitivity (similar to that observed in the EUVL results) with the best dose using the TBAH developer solution observed to be $15.4 \mathrm{~mJ} / \mathrm{cm}^{2}$ when compared with the value of $16.6 \mathrm{~mJ} / \mathrm{cm}^{2}$ with the TMAH developer solution. Note that no significant change was observed in LWR; however, there was an obvious difference in the occurrence of pattern collapse depending on the developer solution. For the TMAH developer solution, pattern collapse was observed at $C D_{\text {collapse }}=38 \mathrm{~nm}$, and, with the sample being developed in the TBAH developer solution, pattern collapse only occurred at $C D_{<\text {collapse }}=32 \mathrm{~nm}$. When compared with the TMAH developer solution, this mitigation of pattern collapse for the ArFi resist is assumed to be caused by the effect of minimized resist swelling during the development process with the TBAH developer solution, as reported in the literature [21]. Using Eqs. (1)-(3) and by replacing $C D_{<\text {breaks }}$

\begin{tabular}{|c|c|c|c|c|c|c|c|}
\hline \multirow{2}{*}{ Developer solution } & \multicolumn{7}{|c|}{ SEM image (CD size $(\mathrm{nm})$ ) } \\
\hline & $\sim 52$ & $\sim 48$ & $\sim 42$ & $\sim 40$ & $\sim 38$ & $\sim 34$ & $\sim 32$ \\
\hline TMAH & & & & & & & \\
\hline $\operatorname{Dose}\left(\mathrm{mJ} / \mathrm{cm}^{2}\right) /$ LWR $(\mathrm{nm})$ & $13.0 / 6.1$ & $14.6 / 5.1$ & $15.8 / 4.9$ & $16.6 / 4.1$ & $17.4 / 3.6$ & $18.6 / *$ & $19 . / *$ \\
\hline TBAH & & & & & & & \\
\hline $\operatorname{Dose}\left(\mathrm{mJ} / \mathrm{cm}^{2}\right) /$ LWR $(\mathrm{nm})$ & $12.2 / 4.9$ & $13.0 / 5.7$ & $14.6 / 4.3$ & $15.4 / 4.0$ & $15.8 / 4.5$ & $17.4 / 3.4$ & $17.8 / 3.8$ \\
\hline
\end{tabular}

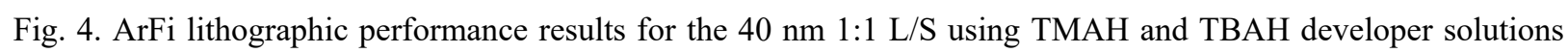
(broken lines indicate optimal dose. Black solid outline indicates absence of pattern bridge or collapse). 


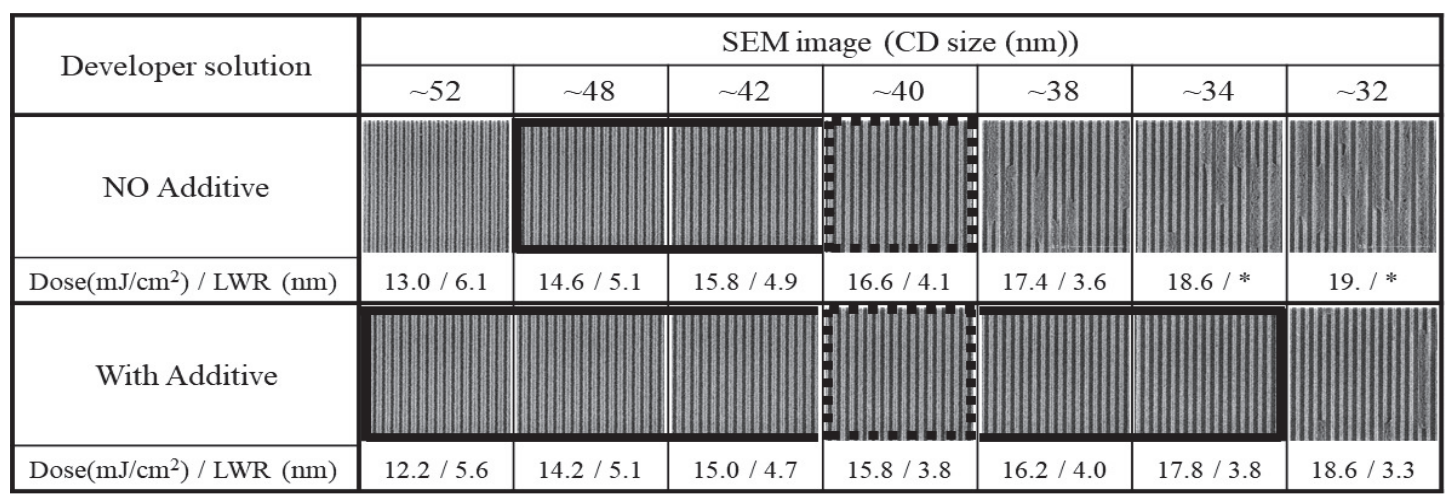

Fig. 5. ArFi lithographic performance results for the $40 \mathrm{~nm} \mathrm{1:1} \mathrm{L/S} \mathrm{using} \mathrm{TMAH} \mathrm{developer} \mathrm{solution} \mathrm{with} \mathrm{and} \mathrm{without}$ additive. Broken lines indicate optimal dose. Black solid outlines indicate absence of pattern bridge or collapse.

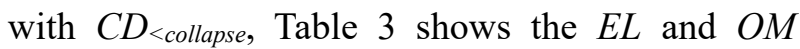
dependence on the developer solution. Significant increases in $E L$ and $O M$ were observed using the TBAH developer solution. Table 4 presents the DNF margin dependence on the developer solution. The results demonstrate a significant improvement in the overdose region using the TBAH developer solution, where the minimum CD size before pattern collapse was $C D_{<\text {collapse }}=34.1 \mathrm{~nm}$ when compared to that obtained with TMAH at $C D_{<\text {collapse }}=39.9 \mathrm{~nm}$. The $C D_{<\text {bridge }}$ values were similar for both the developer solutions, i.e., $\sim 52 \mathrm{~nm}$. This resulted in improved DNF margin when using the TBAH developer solution compared with the usage of the TMAH developer solution, i.e., $43.2 \%$ and $28.6 \%$, respectively.

The results suggest a significant advantage observed while utilizing TBAH as an alternative developer for use in $\mathrm{ArFi}$.

Table 3. ArFi: $E L$ and $O M$ dependence on developer solution type.

\begin{tabular}{c|cc}
\hline $\begin{array}{c}\text { Developer } \\
\text { solution }\end{array}$ & EL (\%) & OM (\%) \\
\hline TMAH & 6.4 & 0 \\
TBAH & 17.3 & 13.0 \\
\hline
\end{tabular}

Table 4. ArFi: DNF margin dependence on developer solution type.

\begin{tabular}{c|ccc}
\hline $\begin{array}{c}\text { Developer } \\
\text { solution }\end{array}$ & $\begin{array}{c}C D_{<\text {bridge }} \\
(\mathrm{nm})\end{array}$ & $\begin{array}{c}C D_{\text {<collapse }} \\
(\mathrm{nm})\end{array}$ & $\begin{array}{c}D N F \text { margin } \\
(\%)\end{array}$ \\
\hline TMAH & 52.9 & 39.9 & 28.6 \\
TBAH & 51.4 & 34.1 & 43.2 \\
\hline
\end{tabular}

3.3 Non-ionic type surfactant additives for ArFi

The application of surfactants during the development and rinse processes is considered to be effective to improve the exposure process margin because it mitigates defects, e.g., pattern collapse
[19]. This increases developer solution hydrophilicity to the resist film, improving the developer solution penetration efficiency.

Figure 5 depicts the ArFi lithographic results for the 40-nm 1:1 L/S pattern obtained using the TMAH developer solutions with and without a surfactant additive. The non-ionic type surfactant was added to the TMAH developer solution at $0.17 \mathrm{wt} \%$, resulting in a developer solution $\mathrm{CA}$ of $40^{\circ}$ (compared to $79^{\circ}$ for the developer solution without additive). Therefore, significant pattern collapse mitigation improvement was achieved, e.g., patterns were observed to have no pattern collapse even at $C D_{<\text {collapse }}=34 \mathrm{~nm}$. The resist sensitivity also improved due to the application of the surfactant additive on the TMAH developer solution (15.8 $\mathrm{mJ} / \mathrm{cm}^{2}$ for the developer solution with the surfactant and $16.6 \mathrm{~mJ} / \mathrm{cm}^{2}$ without the surfactant).

Table 5 presents the ArFi lithographic performance results for the 40-nm 1:1 L/S pattern ( $E L$ and $O M$ dependence on the developer solution with a surfactant additive). The results demonstrate an improvement in both $E L$ and $O M(E L: 16.7 \%$ and OM: $15.8 \%$ using developer solution with a surfactant additive when compared with $E L: 6.4 \%$ and $O M$ : $0 \%$ without any surfactant additive).

Table 6 presents the ArFi lithographic

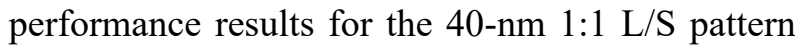
(DNF margin dependence on the developer solution with a surfactant additive).

These results further confirm the effectiveness of the developer solution with a surfactant additive relative to improving the $D N F$ margin, where the minimum CD size achieved before pattern collapse was $C D_{<\text {collapse }}=33.7 \mathrm{~nm}$ when compared with that obtained at TMAH without surfactant at $C D_{<\text {collapse }}$ $=39.9 \mathrm{~nm}$. Here, $C D_{<\text {bridge }}$ improved to $52 \mathrm{~nm}$ for the developer solution with a surfactant additive when compared to $54 \mathrm{~nm}$ without any surfactant additive. These results translate to an approximately 
twice improved DNF margin (51\% using the developer solution with a surfactant additive and $28.6 \%$ without any surfactant additive).

Table 5. ArFi: $E L$ and $O M$ for TMAH developer solution with and without surfactant additive.

\begin{tabular}{c|cc}
\hline $\begin{array}{c}\text { Developer } \\
\text { solution }\end{array}$ & EL (\%) & $O M(\%)$ \\
\hline No Additive & 6.4 & 0 \\
With Additive & 16.7 & 15.8 \\
\hline
\end{tabular}

Table 6. ArFi: DNF margin for TMAH developer solution with and without surfactant additive.

\begin{tabular}{c|ccc}
\hline $\begin{array}{c}\text { Developer } \\
\text { solution }\end{array}$ & $\begin{array}{c}C D_{<\text {bridge }} \\
(\mathrm{nm})\end{array}$ & $\begin{array}{c}C D_{<\text {collapse }} \\
(\mathrm{nm})\end{array}$ & $\begin{array}{c}D N F \\
\text { margin }(\%)\end{array}$ \\
\hline No Additive & 54.1 & 39.9 & 28.6 \\
With Additive & 51.4 & 33.7 & 51.0 \\
\hline
\end{tabular}

\section{Summary}

The application of alternative developers in case of both EUV and ArFi were investigated by focusing on their effects on the DNF margin. In case of EUV lithography, defects were classified as comprising mostly line bridging at the underdose region and line breaks at the overdose region. In case of ArFi lithography, the defects were classified as line bridging at the underdose region and pattern collapse at the overdose region.

The application of alternative developers, such as TBAH, in both the EUV and ArFi lithographic technologies resulted in higher sensitivity when compared with that in TMAH. In case of EUV, this sensitivity improvement was obtained while maintaining the same DNF margin. In contrast, applying the TBAH developer solution to ArFi resulted in a significant reduction of the pattern collapse defects at the overdose region, which translated to a significant increase in the $D N F$ margins. Furthermore, in case of $\mathrm{ArFi}$, the application of a non-ionic surfactant type additive on the TMAH developer solution resulted in resist sensitivity improvement and pattern collapse mitigation, resulting in increased DNF margin.

These results demonstrate the advantages of utilizing TBAH and non-ionic type surfactant additives for improving the DNF margins.

\section{Acknowledgments}

We thank the members of the SCR in AIST for access and technical support in the ArFi tool. We also like to thank the material suppliers for the providing the resist materials and fruitful discussions for this work.

\section{References}

1. R.-H. R. Kim, Proc. SPIE, 10957 (2019) 1095706.

2. E. van Setten, G. Bottiglieri, J. McNamara, J. van Schoot, K. Troost, J. Zekry, T. Fliervoet, S. Hsu, J. Zimmermann, M. Roesch, B. Bilski, and P. Graeupner, Proc. SPIE, 10957 (2019) 1095709.

3. O. Wood II, J. Photopolym. Sci. Technol., 30 (2017) 599.

4. Z. Tasdemir, X. Wang, I. Mochi, L. van LentProtasova, M. Meeuwissen, R. Custers, G. Rispens, R. Hoefnagels, and Y. Ekinci, Proc. SPIE, 10809 (2018) 108090L.

5. D. De Simone, Y. Vesters, P. Venelderen, X. Ran, I. Pollentier, and G. Vandenberghe, Proc. SPIE, 10809 (2018) 108090U.

6. A. Lio, Proc. SPIE, 9776 (2016) 97760V.

7. R. Brainard, Proc. SPIE, 10960 (2019) 1096002.

8. T. Fujimori, T. Tsuchihashi, S. Minegishi, T. Kamizono, and T. Itani, Proc. SPIE, 9776 (2016) 977605.

9. C. Ober, H. Xu, V. Kosma, K. Sakai, and E. P. Giannelis, Proc. SPIE, 10583 (2018) 1058306.

10. A. De Silva, L. Meli, D. Goldfarb, and N. Felix, Proc. SPIE, 10957 (2019) 109570F.

11.P. De Bisschop and E. Hendrickx, Proc. SPIE 10957 (2019) 109570E.

12. R. Fallica, S. J. Rezvani, S. Nannarone, S. Borisov, D. De Simone, S. Babin, G. Lorusso, and G. Vandenberghe, Proc. SPIE, 10960 (2019) 1096009.

13.P. De Bisschop, J. Micro/Nanolith., MEMS MOEMS, 17 (2018) 041011.

14.D. Goldfarb and B. Kumar, Proc. SPIE, 10586 (2018) 1058604.

15. K. Petrillo, G. Huang, D. Ashworth, J. Georger, L. Ren, K-Y. Cho, W. Montgomery, S. Wurm, S. Kawakami, S. Dunn, and A. Ko, Proc. SPIE, 7969 (2011) 796913.

16. R. Gronheid, J. Vac. Sci. Technol. B, 28 (2010) C6S1.

17. M. Harumoto, K. Shigemori. A. Hisai, M. Asai, and K. Kaneyama, Proc. SPIE, 7636 (2010) $76362 Z$.

18. T. Itani and J. J. Santillan, J. Vac. Sci. Technol. B, 27 (2009) 2986.

19. B. W. Smith and K. Suzuki, Microlithography: Science and Technology $2^{\text {nd }}$ edition, CRC Press (2018) 617.

20. S. Shimomura, H. Shimada, R. Au, M. Miyawaki, and T. Ohmi, Proc. SPIE, 1925 (1993) 602.

21.T. Itani and J. J. Santillan, J. Photopolym. Sci. Technol., 23 (2010) 639. 